\title{
Atuação de estudante de enfermagem na campanha de vacinação de covid-19: relato de experiência
}

\author{
Performance of nursing student in the covid-19 vaccination campaign: experience report \\ Desempeño de estudiante de enfermería en la campaña de vacunación covid-19: relato \\ de experiencia
}

Luciana de Carvalho Pires ${ }^{1 *}$, Juliana Silveira Colomé ${ }^{1}$, Carla Lizandra de Lima Ferreira ${ }^{1}$, Daiana Foggiato de Siqueira², Keity Laís Siepmann Soccol ${ }^{1}$.

\section{RESUMO}

Objetivo: Relatar a experiência de estudante de enfermagem na atuação na campanha de vacinação contra a Covid-19. Relato de experiência: Estudo do tipo relato de experiência, desenvolvido a partir da inserção de uma estudante do Curso de Enfermagem, de uma universidade privada, nas campanhas de vacinação contra a Covid-19, em um município do estado do Rio Grande do Sul, no período de março a junho de 2021. A atuação na campanha de vacinação contra a Covid-19 mostrou para a estudante a importância da comunicação, do envolvimento dos voluntários nessas ações, bem como o comprometimento e a responsabilidade social dos estudantes com a população. Pode-se afirmar que a experiência na campanha de vacinação foi de suma importância para o crescimento pessoal e contribuiu para a formação profissional da estudante. Considerações finais: A atuação da estudante evidenciou a importância de um trabalho coletivo e intersetorial para o avanço na vacinação em um momento pandêmico.

Palavras-chave: Pandemias, Saúde pública, Capacitação profissional.

\begin{abstract}
Objective: To report the experience of a nursing student working in the vaccination campaign against Covid19. Experience report: Study of the experience report type, developed from the insertion of a Nursing Course student, from a private university, in the vaccination campaigns against Covid-19, in a municipality in the state of Rio Grande do Sul, in the period from March to June 2021. The performance in the vaccination campaign against Covid-19 showed the student the importance of communication, the involvement of volunteers in these actions, as well as the commitment and social responsibility of students with the population. It can be said that the experience in the vaccination campaign was of paramount importance for personal growth and contributed to the student's professional training. Final considerations: The student's performance highlighted the importance of collective and intersectoral work to advance vaccination in a pandemic moment.
\end{abstract}

Key words: Pandemics, Public health, Professional training.

\section{RESUMEN}

Objetivo: Relatar la experiencia de una estudiante de enfermería que trabaja en la campaña de vacunación contra la Covid-19. Relato de experiencia: Estudio del tipo relato de experiencia, desarrollado a partir de la inserción de una alumna del Curso de Enfermería, de una universidad privada, en las campañas de vacunación contra la Covid-19, en un municipio del estado de Rio Grande do Sul, de marzo a junio de 2021. La actuación en la campaña de vacunación contra el Covid-19 mostró al estudiante la importancia de la comunicación, el involucramiento de los voluntarios en estas acciones, así como el compromiso y responsabilidad social de los estudiantes con la población. Se puede decir que la experiencia en la campaña de vacunación fue de suma importancia para el crecimiento personal y contribuyó a la formación profesional del estudiante. Consideraciones finales: La actuación del estudiante destacó la importancia del trabajo colectivo e intersectorial para avanzar en la vacunación en un momento de pandemia.

Palabras clave: Pandemias, Salud pública, Capacitación profesional.

1 Universidade Franciscana (UFN), Santa Maria - RS. *E-mail: lucianadepires@hotmail.com

2 Universidade Federal de Santa Maria (UFSM), Santa Maria - RS. 


\section{INTRODUÇÃO}

A incorporação do ensino-serviço na formação em saúde possibilita a aprendizagem do aluno próximo da realidade do sistema de saúde e da sociedade na qual convive. Ultrapassar as barreiras físicas das salas de aula e inserir o estudante nos diferentes contextos de prática profissional são métodos para a identificação e compreensão da complexidade das diversas e reais carências dos serviços de saúde, das famílias, das pessoas e da comunidade. Por isso, a formação em saúde deve estar engajada ao processo de ensinoaprendizagem (RODRIGUEZ AM, et al., 2021).

Nesse contexto, faz-se importante lembrar que o processo de ensino-aprendizagem sofreu alterações devido ao contexto da pandemia do novo coronavírus, que perdura desde o ano de 2019 no país. O novo coronavírus, também conhecido como Síndrome Respiratória Aguda Grave 2 (SARS-CoV-2) surgiu em Wuhan, na China, e se propagou por todos os continentes (CONTINI C, et al., 2020). Esse vírus é semelhante ao SARS-CoV no que se refere à sua patogenicidade, evolução clínica e epidemiologia (KANNAN S, et al., 2020).

A pandemia de Covid-19 causou repercussões biomédicas, epidemiológicas e gerou impactos sociais, econômicos, políticos, culturais e históricos sem antecedentes na história das últimas epidemias (DOMINGUES CMAS, 2021). Até o momento, não há informações concretas a respeito da história natural, nem critérios referentes à efetividade para a prática clínica dos casos de infecção humana pelo SARS-CoV2, restando muitos fatores ainda a serem esclarecidos (MINISTÉRIO DA SAÚDE, 2020).

No entanto, alguns países avançaram os estudos sobre a vacinação para imunizar a população o mais rápido possível. Para reduzir os impactos da pandemia, distintos países e empresas farmacêuticas empenharam esforços para produzir vacinas seguras e eficazes contra a Covid-19, bem como no monitoramento daquelas que já se encontram liberadas e registradas em alguns países. No início do ano de 2020, teve início no Brasil a vacinação de alguns grupos prioritários conforme previsto no Plano Nacional de Operacionalização da Vacina contra a Covid-19 (MINISTÉRIO DA SAÚDE, 2021).

Diante do cenário de pandemia de Covid-19 a enfermagem mostrou-se como essencial, ocupando a linha de frente devido a sua atuação, dedicação e competência, bem como a sua atuação intensa se fez relevante nas campanhas de vacinação dos grupos prioritários (CUNHA AG, et al., 2021). Nesse sentido, salienta-se a participação de estudantes de enfermagem nas campanhas de vacinação, com o intuito de somar junto às equipes dos trabalhadores de saúde em prol do avanço na vacinação. Diante do exposto esse estudo teve como objetivo relatar a experiência de estudante de enfermagem na atuação na campanha de vacinação contra a Covid-19.

\section{RELATO DE EXPERIÊNCIA}

Trata-se de um relato de experiência desenvolvido a partir da atuação de uma estudante de enfermagem nas campanhas de vacinação contra a Covid-19. O relato foi construído por meio das vivências da estudante que estava cursando o quarto semestre do curso de graduação em Enfermagem, na disciplina de Saúde Coletiva II, de uma universidade privada do estado do Rio Grande do Sul.

O período do relato compreendeu os meses de março a junho do ano de 2021. A estudante participou oitenta horas da campanha de vacinação, o que permitiu acompanhar a as ações de vacinação de diferentes grupos prioritários. A participação da estudante ocorreu sob supervisão de professores enfermeiros e de enfermeiros da Rede de Atenção à Saúde do município que organizavam a dinâmica da vacinação.

Os locais de vacinação em que a estudante realizou a sua atuação compreendeu espaços sociais como clubes, drive-thru na área urbana, unidades básicas de saúde, estratégias saúde da família, estratégia saúde da família rural, universidade e nos domicílios conforme a necessidade de alguns grupos prioritários, que foram a população de acamados e de seus cuidadores.

Com vistas ao maior entendimento acerca da importância da disciplina de Saúde Coletiva II na matriz curricular do curso de enfermagem, sentiu-se a necessidade de aprofundar o conhecimento a respeito da atuação dos estudantes de enfermagem, de modo voluntário, na vacinação contra o Covid-19. Essa 
experiência foi possível por meio de uma parceria da universidade com a Secretaria Municipal de Saúde, que orientada pelo Ministério da Saúde, deu prioridade à vacinação dos grupos com maior risco à saúde, na tentativa de agilizar as etapas da imunização de toda a população.

Antes da estudante iniciar a participação na Campanha Nacional de Vacinação contra a Covid-19, foram tomadas algumas providências sanitárias em relação à imunização. Inicialmente, a estudante teve um treinamento online ofertado pelos profissionais da Vigilância Epidemiológica do município. Nessa capacitação, a estudante aprendeu sobre os diferentes tipos de imunobiológicos, vias de administração da vacina, condições adequadas de conservação, uso correto de equipamentos de proteção individual e as recomendações padrões sobre a Covid-19.

Ao participar das campanhas de vacinação, a estudante percebeu a importância do esforço coletivo da participação da comunidade acadêmica devido ao grande número de pessoas que aguardavam nas filas para serem vacinadas. Isso, evidenciou a importância da comunicação e da agilidade dos profissionais de saúde para ter um número de voluntários adequado para auxiliarem nas campanhas.

Também, permitiu refletir acerca da importância das instituições de ensino, em incentivar os estudantes para participar dessas ações e assim conhecer os desafios que envolvem a práxis do enfermeiro. Nesse sentido, é evidente a relevância da disciplina de Saúde Coletiva II na formação acadêmica, demostrando a importância da aproximação dos estudantes em ações coletivas.

A atuação na campanha de vacinação contra a Covid-19 mostrou para a estudante a importância da comunicação, do envolvimento dos voluntários nessas ações, bem como o comprometimento e a responsabilidade social dos estudantes com a população. Assim, a mesma compreendeu a importância do retorno social que o seu papel representa para a sociedade.

Também, a estudante percebeu o esforço coletivo na vacinação para atender a demanda de saúde, por isso é necessário que todos tenham subsídios científicos, garantindo com isso um bom desenvolvimento da profissão. Diante disso, verifica-se que há a necessidade de uma base sólida de conhecimento, por isso deve haver a sensibilização do estudante de enfermagem pela busca desse conhecimento, fazendo-se garantir que a vacinação seja segura à população.

Diante do exposto, essa experiência mostrou-se fundamental para o aprendizado e construção do conhecimento, já que foi necessário mobilizar todo o conhecimento adquirido, relacionando as aulas teóricas com a prática. Identificou-se que a atuação na campanha de vacinação foi essencial para o crescimento pessoal e formação profissional da estudante. A atuação da estudante na vacinação evidenciou para a mesma a importância de um trabalho coletivo e intersetorial para que a população fosse vacinada com agilidade e qualidade bem como, aproximou a mesma dos desafios que envolvem a profissão. Ressalta-se, que a experiência foi enriquecedora ao trazer mais conhecimento das normas de biossegurança, garantindo uma experiência profissional única ao contribuir para a saúde da população em meio a um momento pandêmico.

\section{DISCUSSÃO}

Durante a campanha, a estudante questionava as pessoas quanto à manifestação de quadros gripais, períodos de intervalo entre as vacinas e explicava os possíveis efeitos adversos que essas poderiam apresentar. Ainda, reiterava a importância da segunda dose caso aquele imunizante tivesse. Segundo Cunha AG, et al. (2021), é importante explicar aos usuários, antes da aplicação do imunizante, sobre os possíveis efeitos adversos que as pessoas podem apresentar, bem como que cada pessoa pode manifestar efeitos diferentes umas das outras. Desse modo, a estudante orientava sobre a necessidade da busca pela assistência nos serviços de saúde caso os efeitos fossem muito intensos.

As campanhas de imunizações atuam como uma das mais importantes intervenções usadas para o controle de uma doença infectocontagiosa. Corroborando, o Plano nacional de vacinação exige 0 uso de ações inovadoras para superar os desafios do país (WORLD HEALTH ORGANIZATION, 2021; ROCHA TAH, et al, 2021). 
Assim, o uso de metodologias baseadas em inteligência artificial espacial pode fornecer melhorias no planejamento das estratégias de resposta à COVID-19 (ROCHA TAH, et al, 2021). A efetividade dessas práticas só é plausível com um conjunto de competências, como o conhecimento científico acerca dos fatores que influenciam a resposta imune, imunologia, a composição, as interações, a via de administração, as contraindicações das vacinas e os possíveis efeitos adversos esperados pós-vacinação (PÉRICO LAD, WIEDERKEHR PC, 2017; RODRIGUEZ AM, et al., 2021).

Além da preocupação com a realização da técnica de administração do imunizante de modo correto, foi necessária uma comunicação comum entre os profissionais de saúde e as pessoas que queriam se vacinar e os seus acompanhantes, para explicitar as informações de maneira correta, evitando a disseminação das fake news (GALVÃO DN, et al., 2021). Embora, a mídia aborde insistentemente sobre as vacinas nos meios de comunicação, as pessoas ainda apresentam muitas dúvidas sobre a vacinação, principalmente quanto aos efeitos adversos e consumo de bebida alcoólicas após serem vacinadas.

Durante a campanha de vacinação é comum as pessoas demonstrarem diversos sentimentos, mesmo tendo em vista que a vacinação é esperada por uma grande parcela da população (CUNHA AG, et al., 2021). Assim, a estudante percebeu a relevância que o significado da vacinação tem para a população, já que durante a campanha era comum ouvir as pessoas relatando sobre a perda de familiares e amigos antes de ter a oportunidade de terem sido vacinadas.

É notável o nível de aproveitamento do estudante frente à realidade imposta pela pandemia da Covid-19, principalmente no que se refere a oportunidade de somar de forma positiva para a população. Além disso, contribuiu em relação aos conhecimentos sobre a Covid-19 e o aperfeiçoamento dos conhecimentos teóricos e práticos para a formação profissional (PEDREIRA NP, et al., 2021). Ainda, a atuação em campanhas são oportunidades de formar enfermeiros competentes, mostrando-os os desafios que a vida profissional impõe, sobretudo no Sistema Único de Saúde gratuito (PORTUGAL AKJ, et al, 2021).

Atuar em um contexto de pandemia favorece aos estudantes a possibilidade de romper com sentimentos negativos, como insegurança e ansiedade, que interferem no aprendizado e na formação da identidade profissional (SOUZA LB, et al., 2020). Ademais, aponta para a importância do trabalho em equipe e interprofissional. Assim, formam-se enfermeiros sensíveis à importância que o momento requer para que as ações em saúde sejam realizadas com agilidade, qualidade, comprometimento individual e coletivo, potencializando a assistência prestada à população.

Durante a atuação, a estudante observou que o enfermeiro assume um papel fundamental frente às ações de gestão e gerência e no desenvolvimento de ações educativas, haja visto que esses profissionais são os responsáveis por coordenar e organizar cada campanha de vacinação. Isso vai ao encontro de um estudo que evidencia que o enfermeiro assume um papel de destaque nas ações desenvolvidas no Programa Nacional de Imunização, na qual faz a gestão dos imunibiológicos, atua na identificação das situações epidemiológicas, na coordenação da sala de vacinação e na sensibilização das pessoas (GAIVA MAM, 2021). A participação na campanha favoreceu à estudante a compreensão do processo de trabalho do enfermeiro na saúde coletiva, em especial às ações de imunização da população.

Em todas as ações o enfermeiro mostra-se como um protagonista da Atenção Primária à Saúde, sobressaindo desde o planejamento até a execução e avaliação das ações por ele implementadas (RIOS AFM, et al., 2020). Destaca-se o protagonismo desse profissional desde a constituição das comissões, do planejamento e funcionamento da estrutura física, como também pela gestão dos recursos humanos e na elaboração de protocolos e fluxos de cuidado (BITENCOURT JJO, et al., 2020). Nesse sentido, reafirma-se a importância da atuação da enfermagem em salas de vacina, e a capacitação desses profissionais para atuarem frente à pandemia (CUNHA AG, et al., 2021).

Por fim, a experiência em campanhas de vacinação contra a Covid-19 reitera a necessidade do estudante compreender sobre o funcionamento do sistema de saúde público e o conhecimento sobre a vacinação (PEDREIRA et al., 2021). Neste contexto, salienta-se o papel de destaque dos enfermeiros na mobilização da população em favor das vacinas (GAIVA MAM, 2021). 
A enfermagem participa e coopera ativamente com o planejamento e a gestão da logística em setor de imunização no intuito de ofertar uma melhor qualidade e segurança no armazenamento, no manuseio e na distribuição de imunobiológicos. Neste viés, a atuação dessa profissão vai desde o recebimento, averiguação, triagem, armazenamento em temperatura apropriada, preparo, administração das vacinas, descarte adequado dos resíduos. Ainda, compreende o treinamento da equipe, a organização da estrutura de distribuição, o registro de documentos para relatórios, o levantamento de indicadores de desempenho, a prática de auditoria interna e a orientação da população (OLIVEIRA GCA, et al., 2021; COELHO DVCA, et al., 2021).

No panorama da Covid-19, a atuação da Enfermagem tem sido intensivamente na linha de frente no combate à pandemia, tendo ainda, em contrapartida, muitos trabalhadores infectados, outros desenvolvendo algum transtorno psíquico e ainda, correndo o risco de ter suas vidas perdidas. No entanto, o período da pandemia foi oportuno para o reconhecimento da sociedade quanto a importância da atuação da enfermagem nos serviços de saúde, no controle da doença, que mais vez se faz fundamental nesse memento, prestando uma assistência à população com ética, zelo e competência técnica científica (CASTILHO L, 2021).

A atuação da estudante de enfermagem na campanha de vacinação para o novo coronavírus (SARS-CoV2) mostrou-se de extrema importância ao possibilitar a sua inserção na prática profissional e servir como potencializadora do processo formativo da estudante, permitindo relacionar o conhecimento teórico para a aplicação na prática. Assim, evidenciou-se a contribuição da campanha de vacinação para a formação profissional da estudante, também na perspectiva em formar profissionais com conhecimento científico, conhecedor da realidade na prática do cenário de trabalho, e com competências de gestão e gerências em meio à cenários pandêmicos.

\section{REFERÊNCIAS}

1. BITENCOURT JJO, MESCHIAL WC, FRIZON G, et al. Protagonismo do enfermeiro na estruturação e gestão de uma unidade específica para covid-19. Texto \& Contexto Enfermagem, 2020; 29: e20200213.

2. CASTILHO L. A Enfermagem como foco principal ao sucesso da vacinação contra a COVID-19. Revista nursing. 2021; (274): 5344-5345.

3. COELHO DVCA, et al. Experiência do trabalho deuma equipe de Enfermagem na imunização contra a Covid-19 pelo modelo drive-thru. Research, Society and Development. 2021; 10(15); e197101522661.

4. CONTINI C, et al. The novel zoonotic COVID-19 pandemic: an expected global health concern. The Journal of infection in developing countries, 2020; 14(3): 254-64.

5. CUNHA AG, et al. Atuação da enfermagem na campanha de vacinação contra a COVID-19 em um Centro Universitário em Belém-PA. Research, Society and Development, 2021; 10(8): e35310816835.

6. DOMINGUES CMAS. Desafios para a realização da campanha de vacinação contra a COVID-19 no Brasil. Cadernos de Saúde Pública, 2021; 37(1): e00344620.

7. GAIVA MAM. Imunização no contexto da Pandemia de COVID-19. Revista Nursing, 2021; 24(272): 5074.

8. GALVÃO DN, et al. Os desafios durante a campanha de vacinação contra COVID-19: um relato de experiência e reflexões. Research, Society and Development, 2021;10(10): e302101018712.

9. KANNAN S, et al. COVID-19 (Novel Coronavirus 2019) - recent trends. European Review for Medical and Pharmacological Sciences, 2020; 24(4): 2006-2011.

10. MINISTÉRIO DA SAÚDE. Plano Nacional de Operacionalização da Vacina contra a Covid-19. Disponível em: https://www.gov.br/saude/pt br/media/pdf/2021/janeiro/29/PlanoVacinaoCovid_ed4_15fev21_cgpni_18h05.pdf. Acessado em: 06 de janeiro de 2022.

11. MINISTÉRIO DA SAÚDE. Protocolo de Manejo Clínico do Coronavírus (COVID-19) na Atenção Primária à Saúde. Brasília: MS, 2020. Disponível em: https://saude.rs.gov.br/upload/arquivos/202004/14140606-4-ms-protocolomanejo-apsver07abril.pdf. Acessado em: 06 de janeiro de 2022.

12. OLIVEIRA GCA, et al. Assistência de enfermagem no processo de imunização: revisão da Literatura. Brazilian Journal of Development. 2021; 7(1): 7381-7395.

13. PEDREIRA NP, et al. Vivência do acadêmico de enfermagem frente à campanha de vacinação ao combate a pandemia da COOVID-19. Revista Eletrônica Acervo Saúde, 2021; 13(5): e7326.

14. PÉRICO LAD, WIEDERKEHR PC. Imunizações. In: Ferreira SRS, Périco LAD, Dias VRFG. Atuação do enfermeiro na atenção primária à saúde. Rio de Janeiro: Atheneu;2017. p. 243-95.

15. PORTUGAL AKJ, et al. Acadêmicos de enfermagem do interior do Amazonas em missões ribeirinhasdurante a pandemia de covid-19. REAS. 2021; 13(11): 1-6.

16. RIOS AFM, et al. Atenção Primária à Saúde frente à COVID-19: Relato de experiência de um Centro de Saúde. Enfermagem em Foco, 2020; 11(1esp): 246-25.

17. ROCHA TAH, et al. Plano nacional de vacinação contra a COVID-19: uso de inteligência artificial espacial para superação de desafios. Ciência \& Saúde Coletiva. 2021; 26(5): 1885-1898.

18. RODRIGUEZ AM, et al. Vacinação contra influenza no enfrentamento da Covid-19: integração ensino-serviço para formação em enfermagem e saúde. Escola Anna Nery Revista de Enfermagem, 2021; 25(esp): e20200379.

19. SOUZA LB, et al. Estágio curricular supervisionado em enfermagem durante a pandemia de Coronavírus: experiências na atenção básica. Journal of nursing and health, 2020; 10 (esp): e20104017.

20. WORLD HEALTH ORGANIZATION. Best practices in microplanning for polio eradication. Geneva: WHO; 2021. 56 p. 